\title{
Early Aggressive Treatment Approaches for Multiple Sclerosis
}

\author{
Alexandra Simpson, $M D^{1}$ \\ Ellen M. Mowry, MD, MCR \\ Scott $D$. Newsome, $D 0^{1,2, *}$
}

\author{
Address \\ ${ }^{1}$ Department of Neurology, Johns Hopkins School of Medicine, Baltimore, MD, USA \\ ${ }^{*}, 2$ Division of Neuroimmunology and Neurological Infections, Johns Hopkins \\ Hospital, 600 North Wolfe St., Pathology 627, Baltimore, MD, 21287, USA \\ Email: snewsom2@jhmi.edu
}

Published online: 15 May 2021

(C) The Author(s), under exclusive licence to Springer Science+Business Media, LLC, part of Springer Nature 2021

This article is part of the Topical Collection on Multiple Sclerosis and Related Disorders

Keywords Multiple sclerosis - Relapsing-remitting multiple sclerosis - Disease-modifying therapy · High-efficacy therapies

\section{Abstract}

Purpose of review This review presents a comprehensive analysis of the current highefficacy disease-modifying therapies (DMTs) available for treatment of multiple sclerosis (MS). We discuss the existing approved and emerging therapeutics in patients with relapsing and progressive forms of MS using data from clinical trials and observational studies. Treatment considerations in pediatric and pregnant populations are also reviewed. Finally, we discuss the treatment paradigms of the escalation and early aggressive approaches to treatment of MS, with review of ongoing clinical trials to compare these approaches.

Recent findings Natalizumab has shown promising data on efficacy in not only randomized trials but also observational studies when compared with placebo, the injectable DMTs, and fingolimod. The anti-CD20 B cell depleting therapies (rituximab, ocrelizumab, and ofatumumab) have also demonstrated superiority in randomized clinical trials compared to their comparator group (placebo, interferon, and teriflunomide, respectively) and rituximab has shown in observational studies to be more effective than older injectable therapies and some of the oral therapies. Alemtuzumab has shown good efficacy in randomized controlled trials and observational studies yet has several potentially severe side effects limiting its use. Mitoxantrone has similarly demonstrated significant reduction in new disease activity compared to placebo but is rarely used due to its severe side effects. Cladribine is an oral DMT often grouped in discussion with other higher efficacy DMTs but may be slightly less effective than the other therapies described in this review. 
Many emerging targets for therapeutic intervention are currently under investigation that may prove to be beneficial in early aggressive MS, including autologous hematopoietic stem cell transplantation.

Summary Traditionally, MS has been treated with an escalation approach, starting patients on a modestly effective DMT and subsequently escalating to a higher efficacy DMT when there is evidence of clinical and/or radiologic breakthrough activity. With the development of higher efficacy therapies and emerging data showing the potential positive longterm impact of these therapies when started earlier in the disease course, many clinicians have shifted to an early aggressive treatment approach in which patients are initially started on a higher efficacy DMT. Two clinical trials, the TRaditional versus Early Aggressive Therapy for MS (TREAT-MS) trial and the Determining the Effectiveness of earLy Intensive Versus Escalation approaches for the treatment of Relapsing-remitting MS (DELIVER-MS) trial, aim to directly compare these treatment strategies and their impact on clinical and radiologic outcomes.

\section{Introduction}

Multiple sclerosis (MS) is the most common neuroinflammatory disorder of the central nervous system. The immune system plays a significant role in the pathogenesis of MS; thus, the development of immunomodulating/immunosuppressive therapies has been critical for treating MS. The expanding armamentarium of therapeutics has helped alter the clinical course of MS with its impact on minimizing inflammatory disease activity.

A more aggressive course of MS has been associated with a variety of clinical and paraclinical factors. Patients who are male and African American/Black or Hispanic, have a later age at onset, have frequent relapses with poor recovery (particularly with motor, cerebellar, and sphincter involvement), or have evidence of early cognitive dysfunction appear to be at risk of more disabling phenotypes of MS [1-6]. Moreover, high lesion burden on magnetic resonance imaging (MRI), lesion location (brainstem, cerebellum, spinal cord), development of new lesions in the first few years after symptom onset, early optical coherence tomography (OCT) changes, and presence of oligoclonal bands can increase the risk of more aggressive MS [7-10]. However, the initial absence of these factors does not necessarily translate to a benign phenotype long-term, and studies are needed that include putative prognostic factors evaluated concomitantly, appropriately accounting for disease-modifying therapy use/strategy in more representative cohorts. An observational study by Leray et al. [11] followed 874 patients for at least 10 years and found that initial clinical factors associated with a more favorable outcome did not predict a benign course of disease longterm with each decade that passed.

There are different treatment approaches in MS and specifically in relapsing MS. At this juncture, it is not clear what treatment approach is best especially early on in MS. Traditionally, the escalation treatment approach has been utilized and is still common practice among clinicians. With this approach, patients are started on disease-modifying therapies (DMTs) with moderate efficacy on inflammatory activity and remain on this DMT until there is evidence of new clinical or radiologic activity. However, with the advances in treatment of MS, there are now several agents that appear to have a greater impact on inflammation (classified as high efficacy) compared to moderately effective therapies. Hence, there has been a lot of interest in recent years to consider starting high-efficacy treatments earlier in MS, also known as the early aggressive approach [12]. The hypothesis behind starting highly efficacious therapies early in the disease course is that such an approach may maximize the potential for preventing disability progression over time.

There is a paucity of evidence-based guidelines for determining which treatment strategy/approach is best for patients with MS. While clinical trials have shown that the efficacy of MS therapies varies, this does not necessarily translate to the same differences in realworld effectiveness. Some insights into real-world effectiveness have been gained by observational studies, 
short randomized controlled trials, meta-analyses, and comparative effectiveness research, but even these present limitations. This review will focus on pivotal studies of current and emerging DMTs in the treatment of MS, with an emphasis on early aggressive treatments in MS. Additionally, we will discuss gaps in knowledge as well as ongoing pragmatic trials assessing treatment strategies in relapsing MS.

\section{Treatments}

Basic principles of multiple sclerosis disease-modifying therapies

- Starting treatment shortly after initial symptom onset gives patients the

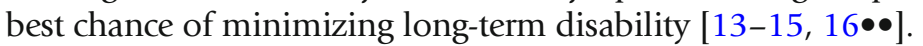

- Having a low threshold to switch therapies when there is breakthrough disease activity (clinical relapses and/or new lesions on MRI) may help prevent future disability [17].

- If a patient has an inadequate (subtherapeutic) treatment response to a DMT, it is prudent to choose another DMT with a different mechanism of action $[13,18]$.

- Infusion therapies, cladribine, and ofatumumab appear to be more effective than the other therapies, although some experts consider sphingosine1-phosphate inhibitors to be intermediate or higher efficacy [19-21, 22•, 23].

\section{Higher efficacy disease-modifying therapies}

The classification of a DMT as higher efficacy occurs based on favorable results from clinical trials comparing that treatment to placebo or traditional DMTs such as interferon beta or glatiramer acetate. The annualized relapse rate (ARR) and progression of disease (often measured using the EDSS) are commonly used clinical outcomes in MS research, but MRI and other paraclinical data are also increasingly being used to study drug efficacy. A combined endpoint outcome measure that is utilized in studies is "no evidence of disease activity" (NEDA). NEDA is characterized by lack of clinical relapses, no new or enlarging lesions on MRI, and absence of disability progression. A DMT is labeled as higher efficacy when the data show a more favorable treatment impact on at least the inflammatory outcome (relapses and new lesions), although some experts also prefer to evaluate lack of disability progression in considering relative efficacy.

Randomized, placebo-controlled clinical trials are traditionally the gold standard for conducting clinical research and provide important data on a drug's efficacy. It is imperative to note that efficacy in clinical trials occurs in ideal and controlled conditions and thus may not necessarily translate to the real world, where observational and comparative effectiveness studies as well as pragmatic trials are better able to provide an understanding of relative effectiveness. Comparisons across clinical and observational trials are difficult, given the patient populations in each study can vary significantly. Additionally, it is often difficulty to apply group-level data to an individual. Furthermore, group-level mean differences in outcomes between treatment arms in a clinical trial are 
often driven by subsets of the patients in each arm, and thus, it can be difficult to apply the data from a group to an individual patient in the real world.

Many consider the higher efficacy medications to include infusions (natalizumab, alemtuzumab, rituximab, ocrelizumab, and mitoxantrone), oral cladribine, and subcutaneous ofatumumab, although there is some discussion on whether additional therapies, in particular the sphingosine-1-phosphate receptor modulators, should be included under this label. The mechanism, dosing, clinical trial and observational data, and safety concerns will be addressed for each treatment.

\section{Infusion therapies and ofatumumab}

\section{Natalizumab}

Natalizumab is a monoclonal antibody directed against the alpha-4 subunit of integrin which helps to block lymphocyte entry into the central nervous system. The standard dosing is $300 \mathrm{mg}$ administered intravenously every 4 weeks, although extended interval dosing (EID) has been implemented to mitigate risk of progressive multifocal leukoencephalopathy (PML) in patients with positive serum John Cunningham virus (JCV) antibodies. A respective cohort study analyzing risk of PML in 35,521 JCV antibody-positive patients treated with natalizumab in an average 30-31-day standard interval dosing (SID) or average 35-43-day EID showed a $94 \%$ and $88 \%$ relative risk reduction in developing PML with EID in the primary and secondary analyses, respectively [24].

In the original randomized, placebo-controlled trial, the Natalizumab Safety and Efficacy in Relapsing-Remitting Multiple Sclerosis (AFFIRM) study, 942 patients with relapsing MS were randomly assigned to receive natalizumab or placebo. Natalizumab demonstrated a $42 \%$ relative risk reduction (RRR) in disability progression at 2 years and $68 \%$ reduction in the ARR at 1 year when compared to placebo. Secondary MRI outcomes showed a reduction in number of T2-weighted lesions by $83 \%$ and reduction in gadolinium-enhancing lesions by $92 \%$ compared to placebo [25]. The REVEAL study was a phase 4 active comparator clinical trial comprising 108 patients with relapsing MS randomized to receive either natalizumab or fingolimod in a 1:1 fashion. The primary endpoint was the progression of new on-treatment gadolinium-enhancing lesions to persistent black holes on MRI at 52 weeks. Due to early study termination, the primary endpoint could not be assessed. Unplanned exploratory analyses of secondary outcomes revealed the mean number of new gadolinium-enhancing lesions was at least 70\% lower at 12 and 24 weeks in natalizumab-treated patients, while other MRI outcomes of new or enlarging T2 lesions were not significant between groups. With regard to clinical outcomes, the ARR was $90 \%$ lower in patients treated with natalizumab than with fingolimod and the cumulative probability of relapse was lower in the natalizumab-treated group [26].

Subsequent observational studies have evaluated the effectiveness of natalizumab when compared with injectable DMTs and fingolimod. In a study using propensity score methods, in 732 treatment-naïve patients receiving firstline natalizumab or injectable DMTs (interferon beta (IFN- $\beta$ ) or glatiramer acetate (GA)), natalizumab was associated with a $68 \%$ relative reduction in ARR compared with IFN- $\beta /$ GA [27]. Natalizumab has also been favored as an 
option in escalation of treatment after patients demonstrate inadequate response to treatment with traditional injectable medications. An observational study by Kalincik et al. used longitudinal data obtained from 792 patients in an MS patient registry to compare effectiveness of starting natalizumab versus fingolimod after treatment failure with IFN- $\beta /$ GA. The ARR declined from 1.5 to 0.2 in patients treated with natalizumab, compared with reduction in ARR from 1.3 to 0.4 in patients treated with fingolimod, corresponding to a relative $50 \%$ lower relapse rate with initiation of natalizumab than fingolimod [20]. Additionally, the rate of sustained disability regression was 2.8 times higher in natalizumab-treated patients than in fingolimod-treated patients. Patients with relapses on glatiramer acetate and interferons are shown to have fewer relapses and higher rates of NEDA when escalating to natalizumab compared to those switching to fingolimod or remaining on injectable therapies $[28,29]$. These data suggest that an escalation in treatment rather than lateral switch to another injectable therapy results in more favorable patient outcomes.

Even though the efficacy data for natalizumab appear superior to traditional therapies, it does not mean that every patient is a candidate for or will need natalizumab. It is important to consider the potential risks associated with natalizumab and other high-efficacy therapies (see below under respective therapies) prior to starting or switching to these therapies. Some contraindications to the use of natalizumab include a history of progressive multifocal leukoencephalopathy (PML) and infusion reactions that are related to natalizumab neutralizing antibodies. Although, it is also generally recommended to not use natalizumab in patients with high serum JCV antibody titers (> 0.90 ) and, given the availability of other higher efficacy medications, even those with a lower, albeit positive, JC virus antibody titer may want to consider other options or consider EID options if they do start it. Major side effects include $\mathrm{PML}$, infusion-related reactions, liver failure (autoimmune hepatitis), herpes

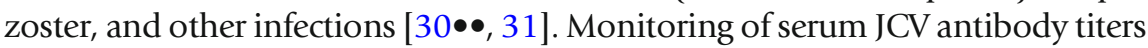
should occur approximately every 3-6 months which in part depends on patient's serostatus. Providers should evaluate routine brain MRIs for radiologic findings concerning for PML. Additionally, natalizumab infusions should be delayed for any new symptom concerning for PML while obtaining an MRI for diagnosis.

Anti-CD20 B cell depleting therapies: rituximab, ocrelizumab, and ofatumumab

\section{Rituximab}

Rituximab is a monoclonal antibody in the category of $\mathrm{B}$ cell depleting therapies targeting the CD20 antigen on B cells. Historically, rituximab has been used more often in treatment of neuromyelitis optica spectrum disorder (NMOSD) in the United States (US) but has higher rates of use in European nations for MS. While rituximab has been used off-label in the US for treatment of MS based on data from phase 2 trials, it does not have formal FDA approval for this indication. Traditionally, initial dosing of rituximab consists of a 1000-mg dose on day 1 , followed by a second 1000-mg dose on day 15, and repeating this regimen every 6 months. Although, many MS specialists change the traditional dosing regimen after patients have been on rituximab for several years to help 
mitigate the risk of long-term infections which is discussed in more detail below.

In a phase 2, double-blind, 48-week trial in 104 patients with relapsingremitting MS (RRMS) randomized to rituximab or placebo, treatment with rituximab showed a $91 \%$ relative risk reduction in number of total contrastenhancing lesions on MRI when compared to placebo. Rituximab was also associated with fewer clinical relapses at 48 weeks, with $20.3 \%$ of patients in the rituximab group experiencing relapses versus $40.0 \%$ of patients in the placebo group [32]. Rituximab has also been studied in primary progressive MS (PPMS). A randomized double-blind, placebo-controlled multicenter trial of 439 patients with PPMS did not show clinical significance between time to confirmed disease progression between the two groups or the secondary endpoint of brain volume change. However, rituximab showed favorable results in the secondary endpoint of T2 lesion volume and improvement in the timed 25 -ft walk test [33]. Moreover, in the subgroup analysis, the younger patients ( $<51$ years) with gadolinium-enhancing lesions on MRI subgroup demonstrated a clinically significant increase in the time to confirmed disease progression (primary outcome) when treated with rituximab versus placebo.

There are several recent observational studies from Sweden highlighting rituximab's effectiveness in MS. In a study comparing outcomes for $256 \mathrm{MS}$ patients transitioning from natalizumab to rituximab or fingolimod following JCV seropositivity, patients treated with rituximab had favorable outcomes across several measures. Patients switched to rituximab had fewer clinical relapses $(1.8 \%$ compared to $17.6 \%$ with fingolimod), fewer contrastenhancing lesions on MRI (1.4\% compared to $24.2 \%)$, less risk of discontinuation (1.8\% compared to $28.2 \%$ ), and fewer adverse events (5.3\% compared to $21.1 \%$ ) at 1.5 years after treatment switch from natalizumab compared to fingolimod [34]. In a comparative effectiveness study of 494 patients' initial treatment with rituximab, injectable DMTs, dimethyl fumarate, fingolimod, or natalizumab, rituximab was superior to dimethyl fumarate and injectable DMTs with regard to rate of clinical relapses, radiologic disease activity, and discontinuation rate [35]. In fact, rituximab had the most favorable discontinuation rate (0.03) of all DMTs. While treatment failure was the most common reason for discontinuation of injectable DMTs (0.53), dimethyl fumarate (0.32), and fingolimod (0.38), JCV antibody-positive status was the main reason for discontinuation of natalizumab (0.29).

Ocrelizumab is a recombinant humanized IgG monoclonal antibody directed against the CD20 antigen located on the cell surface of B-lymphocytes. It is one of a few B cell depleting therapies that have been used in treating MS. This therapy is initiated with a 300-mg infusion, followed by a second 300-mg infusion 2 weeks later, and subsequent 600 -mg dosing every 6 months thereafter [36].

The OPERA I and II phase 3 clinical trials randomized 821 and 835 patients with relapsing forms of MS to receive ocrelizumab or interferon beta-1a three times a week. Patients treated with ocrelizumab had an at least $46 \%$ reduction in the annualized relapse rate at 96 weeks compared to patients on interferon therapy and slowed disability progression at 12 and 24 weeks by 33\% and 34\%, 
respectively. Patients on ocrelizumab also showed at least 94\% reduction in the number of new gadolinium-enhancing lesions on MRI in comparison to interferon-treated patients [37].

In addition to its use in relapsing MS, ocrelizumab was the first FDAapproved medication for use in treatment of primary progressive MS based on results from the randomized, double-blind, placebo-controlled ORATORIO study. In this clinical trial of 732 PPMS patients, patients on ocrelizumab showed less disability progression than patients on placebo at 12 (32.9\% versus $39.3 \% ; p=0.03$ ) and 24 weeks (29.6\% versus $35.7 \% ; p=0.04)$. Additionally, ocrelizumab-treated patients had a decrease in the volume of brain lesions and percentage of brain volume loss on T2-weighted MRI compared to the placebo group [38].

\section{Ofatumumab}

Ofatumumab is a monoclonal antibody directed against the CD20 antigen on the surface of B cell lymphocytes, with a greater effect on cells by complementdependent cytotoxicity over antibody-dependent cellular cytotoxicity [39]. It is given as a 20-mg subcutaneous injection monthly. In the phase 2 MIRROR study, ofatumumab demonstrated a less substantial effect on B cell depletion with shorter duration to recovery of B cell populations than what has previously been observed with other intravenous B cell-targeted therapies.

The phase 3 double-blind ASCLEPIOS I and II clinical trials randomized a total of 1882 patients to receive either ofatumumab or teriflunomide for up to 30 months, with a primary endpoint of annualized relapse rate. In ASCLEPIOS I and II, the ARR was reduced by $51 \%$ ( 0.11 vs. 0.22$)$ and $58 \%$ ( 0.10 vs. 0.25$)$ in favor of ofatumumab, respectively [23]. Additionally, secondary MRI endpoints revealed a significant decrease in the mean number of gadolinium-enhancing lesions on MRI (94-97\%) and an $82-85 \%$ reduction in the mean number of new or enlarging T2 lesions per year with ofatumumab compared to teriflunomide. There were no significant differences between treatment groups in annualized rate of brain volume loss. In pooled analyses from both trials, ofatumumab showed less worsening of disability at 3 and 6 months than teriflunomide, but there were no significant differences between groups in disability improvement at 6 months. Finally, ofatumumab was associated with lower serum neurofilament light chain concentrations.

\section{Risk profile and monitoring in anti-CD20 B cell depleting therapies}

The risks and safety profile of the anti-CD20 B cell depleting therapies will be discussed together given their similarities in mechanism of action. While ocrelizumab is associated with more antibody-dependent cell-mediated cytotoxicity (ADCC) and less complement-dependent cytotoxicity (CDC) than rituximab or ofatumumab [40], the implications of these differences and their effects on development of certain infections have not been clearly delineated. The most common side effects of this class are infusion or injection-site reactions, while more severe adverse events include bone marrow suppression, reactivation of viruses (hepatitis $\mathrm{B}$ and herpes zoster), tuberculosis, PML, and possible development of certain malignancies [37, 41, 42]. In 2011, a case series was published on 4 cases in addition to the one previously known case of PML associated with rituximab use in treatment of rheumatoid arthritis (RA) [43]. 
There has been one confirmed case of PML in an elderly patient on ocrelizumab to date. While development of PML remains a potential risk in MS patients treated with anti-CD20 B cell depleting therapies, this risk remains rare. A Swedish cohort study of infection risks in 6421 patients treated with rituximab, natalizumab, fingolimod, interferon beta, or glatiramer acetate revealed the highest rate of serious infections with rituximab (hazard ratio 1.7). There are also data suggesting that anti-CD20 therapies may be associated with increased risk of SARS-CoV-2 infection, and that infection severity may be greater in those treated with anti-CD20s for a longer period of time [44]. Regarding screening and routine lab monitoring including complete blood cell count (CBC) with differential, liver function tests (LFTs), and hepatitis panel, refer to the prescribing information for complete information.

Most neurologists have reduced the dosage used of rituximab to as little as a single infusion of $500 \mathrm{mg}$, and many clinicians consider reducing the dose frequency as well, particularly over time, in order to reduce the risk of infection with long-term use. Similar to rituximab, some clinicians are recommending using extended interval dosing and/or 300-mg over 600-mg dosing of ocrelizumab in patients after a few years. The impact of these changes on effectiveness remains to be demonstrated in well-controlled comparative effectiveness studies or clinical trials.

\section{Alemtuzumab}

Alemtuzumab is a monoclonal antibody directed toward the CD52 surface antigen on B- and T-lymphocytes, macrophages, and other white blood cells. Dosing consists of an IV infusion of $12 \mathrm{mg}$ daily for 5 consecutive days in year 1 , followed by $12 \mathrm{mg}$ daily for 3 consecutive days in year 2 and beyond (if deemed necessary).

The CARE-MS I and II randomized controlled trials were the pivotal studies demonstrating alemtuzumab's use in MS. In CARE-MS I, 563 previously untreated patients with RRMS were randomized to receive either alemtuzumab or interferon beta-1a three times a week. Subjects receiving alemtuzumab had a reduction in relapses of $54.9 \%$ compared to those treated with interferon beta1a. Alemtuzumab also showed fewer new or enlarging T2 lesions (48\% compared with 58\% in interferon group), and fewer gadolinium-enhancing lesions at 24 months $(7 \%$ in alemtuzumab arm compared with $19 \%$ in interferon group) [45]. The CARE-MS II trial also compared rates of efficacy and adverse events with alemtuzumab and interferon beta-1a three times a week, but in 798 patients with RRMS who had failed first-line therapies. Alemtuzumab reduced the relapse rate by $49.4 \%$ and resulted in fewer new or enlarging T2 lesions (46\% vs $68 \%$ ) or contrast-enhancing lesions (9\% vs $23 \%$ ) on MRI when compared to interferon beta-1a [46]. The extension studies have shown that many patients from the core study have required no further treatment after the 2 courses of treatment and have consistently achieved NEDA in each year.

A comparative effectiveness study by Kalincik et al. [47] used propensity score matching to compare relapse rates and disability in 4332 RRMS patients from various cohorts treated with alemtuzumab, interferon beta, fingolimod, or natalizumab. The primary endpoint was ARR, and treatment with alemtuzumab was associated with a lower ARR than both interferon beta and fingolimod, with a similar ARR compared to natalizumab. Primary analysis of 
secondary endpoints showed similar probability of disability accumulation and improvement between all groups. Secondary analyses revealed alemtuzumab had a lower probability of disability accumulation than interferon beta in patients with more active disease, and a higher probability of disability improvement in patients with prior on-treatment relapses. Natalizumab was associated with a higher probability of disability improvement than alemtuzumab.

Alemtuzumab is contraindicated in HIV-positive patients. Infusion-related reactions and lymphopenia are the most common side effects of alemtuzumab. There are several potential severe side effects that can occur with alemtuzumab that are important to recognize early including bone marrow suppression, antiglomerular basement membrane disease, thyroid disease, stroke and arterial dissection, infections (Listeria monocytogenes, herpes zoster, PML, etc.), and malignancies (melanoma, thyroid cancer, and lymphomas) to name a few [48]. While alemtuzumab is typically reserved for treatment of highly active MS in the US, it is used more frequently in the UK, making comparisons of prescribing differences between alemtuzumab and other high-efficacy therapies difficult.

\section{Mitoxantrone}

Mitoxantrone is an anthracenedione that acts as an intercalating agent, resulting in DNA cross-links and strand breaks. It is approved for use in MS as well as some cancers. It is administered as an intravenous infusion $12 \mathrm{mg} / \mathrm{m}^{2}$ every 3 weeks for up to 10 cycles. A maximum cumulative lifetime dosing of $140 \mathrm{mg} /$ $\mathrm{m}^{2}$ has been established based on the significant risk profile.

One randomized placebo-controlled clinical trial involving 51 RRMS patients showed a $66 \%$ reduction in the yearly relapses in a mitoxantrone-treated group when compared to placebo. The primary outcome of progression of disability, or increase in EDSS scores by more than 1 point, was also found to be lower in mitoxantrone than in placebo groups in the first year; however, this trend did not become statistically significant until year 2 of the study. Outcomes related to MRI imaging showed no difference between the number of new or enlarging lesions [49]. Mitoxantrone has also been studied in secondary progressive multiple sclerosis, with one placebo-controlled trial including 194 SPMS and worsening RRMS patients demonstrating a $66 \%$ reduction in ARR in mitoxantrone-treated subjects in addition to improvement in multivariate analysis of multiple clinical variables in the treatment group [50].

Mitoxantrone has significant side effects associated with its use, including myelosuppression, cardiotoxicity resulting in heart failure, and acute myeloid leukemia [51]. Some of these side effects are not dose-dependent. Despite the high efficacy of the drug, its use has fallen out of favor due to the risk of severe and potentially fatal adverse events.

\section{Oral therapies}

\section{Cladribine}

Cladribine is an infrequently administered oral DMT that is found to be highly effective in the treatment of MS, although it may be slightly less effective than the aforementioned highly efficacious therapies. Cladribine is delivered in a cumulative dose of $3.5 \mathrm{mg} / \mathrm{kg}$ over 2 years of treatment, broken up into two 
treatment courses (each $1.75 \mathrm{mg} / \mathrm{kg}$ ) spread over 2 months each year.

The CLARITY study was a 96-week randomized placebo-controlled phase 3 trial of the efficacy and safety of cladribine use in 1326 RRMS patients. The primary endpoint was the annualized relapse rate at 96 weeks, and patients treated with the current FDA-approved dose of cladribine were shown to have a $58 \%$ reduction in annualized relapse (0.14) compared with patients in the placebo group (0.33), but notably patients in the $3.5 \mathrm{mg} / \mathrm{kg}$ cladribine group had a shorter mean duration of disease than the placebo and $5.25 \mathrm{mg} / \mathrm{kg}$ cladribine groups [52]. Additionally, patients treated with cladribine had higher rates of remaining relapse-free, longer durations to first relapse, and less disease activity on MRI (marked by fewer gadolinium-enhancing lesions, 86\%). A post hoc analysis of the CLARITY study showed a $47 \%$ reduction in the risk of sustained disability progression over 6 months in patients treated with cladribine versus placebo (47\%) [53].

One observational study used propensity score matching and analysis to compare cladribine to other DMTs, merging data from 945 patients in the CLARITY trial and 2204 patients in an Italian MS patient database. Patients treated with cladribine had a significant reduction in the ARR when compared with interferon therapy (52\%), glatiramer acetate (51\%), and dimethyl fumarate $(40 \%)$. There was no statistically significant difference in ARR between cladribine and fingolimod. Natalizumab had a more favorable outcome, showing a reduction in the ARR by $53 \%$ when compared to cladribine. A subgroup analysis revealed cladribine reduced the ARR by approximately $70 \%$ in patients with high disease activity (defined as $\geq 2$ relapses the year prior to study) versus interferon, glatiramer acetate, and dimethyl fumarate [54]. A meta-regression analysis comparing data on efficacy of cladribine, fingolimod, natalizumab, alemtuzumab, and ocrelizumab showed comparative relative efficacy in reducing the ARR compared with placebo, with a matching-adjusted indirect comparison demonstrating comparable efficacy between cladribine and alemtuzumab [55].

Common side effects include fatigue and headache. More severe side effects include risk of myelosuppression, opportunistic infections, nephrotoxicity, and possible increased risk of malignancy. Cladribine is contraindicated in patients with active malignancy and in patients who are or wish to become pregnant during the treatment course due to its teratogenic effect [56].

\section{Putative emerging highly efficacious interventions}

\section{Autologous hematopoietic stem cell transplantation}

Autologous hematopoietic stem cell transplantation (AHSCT) has been a topic of strong interest in the MS community. Ablation of an individual's immune system with chemotherapy and/or broad immune suppressant therapies followed by AHSCT has been studied in various autoimmune diseases, and several studies in the past decade have suggested its efficacy in certain subtypes of MS. There are two widely used options for conditioning regimens, the intermediate-intensity myeloablative BEAM-ATG (carmustine, etoposide, cytarabine-arabinoside, melphalan, and anti-thymocyte globulin (ATG)) regimen, and the intermediate-intensity nonmyeloablative regimen consisting of cyclophosphamide and ATG [57]. 
One observational study of 281 patients (78\% PPMS or SPMS, 22\% RRMS) treated with AHSCT showed participants had a $46 \%$ probability of achieving progression-free survival in the 5 years following transplantation, with EDSS progression defined as an increase in 1 point on the EDSS at 12 months compared to baseline [58]. More favorable outcomes were found in patients with relapsing MS, younger age, lower initial EDSS score, and fewer treatment with prior immunotherapies. With multivariate analysis, only higher baseline EDSS was associated with worse survival over time. While AHSCT is not yet approved by the FDA for treatment of MS, the American Society for Blood and Bone Marrow Transplantation has deemed AHSCT an appropriate treatment for patients with relapsing MS who have proven refractory to high-efficacy conventional therapies and have the potential for disability accumulation due to active disease [59]. AHSCT is associated with risks much like those expected in other forms of transplantation. One study suggested that, while AHSCT can be highly effective in suppressing inflammatory disease activity, there is an increase in whole brain atrophy attributed to treatment-related toxicity [60]. The BEAT-MS (Best Available Therapy Versus Autologous Hematopoietic Stem Cell Transplant for Multiple Sclerosis) study is a 6-year ongoing study currently investigating AHSCT versus high-efficacy DMTs (natalizumab, alemtuzumab, ocrelizumab, or rituximab) with a primary endpoint of relapse-free survival up to 36 months [61]. This study may provide the necessary evidence for demonstrating the potential use of AHSCT in specific subgroups of MS patients. It is yet to be determined whether the short-term benefits of AHSCT will outweigh risks, or whether the use of AHSCT may limit a patient's potential use of other DMTs in the future.

\title{
Additional therapies under investigation
}

The rapid expansion of MS therapeutics is ongoing, with many investigative therapies currently in various phases of clinical trials. For example, ublituximab is a monoclonal antibody targeting a specific epitope on the CD20 antigen, with an antibody-dependent cellular cytotoxicity mechanism of action targeting B cells. A phase 2 multicenter, placebo-controlled trial demonstrated promising results, with median B cell depletion $>99 \%$ at week 4 (maintained at 24 and 48 weeks), no gadolinium-enhancing lesions at 24 and 48 weeks, and decrease in size of T2 lesions at 24 and 48 weeks. Overall, 93\% of patients remained relapse-free during the study, with $74 \%$ of patients achieving NEDA [62]. Another interesting class of medications include Bruton's tyrosine kinase (BTK) inhibitors. There appears to be an impact on both adaptive and innate immune cells with BTK inhibitors which could prove to be very effective. A phase 2 double-blind, randomized trial showed that patients treated with evobrutinib, an oral BTK inhibitor, had significantly fewer total contrasting-enhancing lesions at 12 through 24 weeks than patients in the placebo group [63].

\section{Special considerations}

\author{
Pediatric considerations
}

Limited studies exist on the use of highly effective DMTs for treatment of pediatric MS, stemming from the small population of patients $(\sim 5 \%)$ and concerns for impact on development with initiation of many DMTs. 
Historically, interferon beta and glatiramer acetate have been preferred firstline therapies due to their long history of use and acceptable safety profiles [64]; however, the use of more effective DMTs in pediatric populations is increasing [65]. In one phase 3 trial of 215 pediatric MS patients receiving fingolimod or interferon beta-1a, fingolimod-treated patients showed an $82 \%$ reduction in the ARR and 53\% reduction in the annualized rate of new or newly enlarged lesions on T2-weighted MRI when compared with interferon-treated patients [66]. In patients who have highly active disease refractory to interferon beta and glatiramer acetate, natalizumab has been the preferred second-line agent. In one study using an Italian registry of 101 pediatric patients with MS who received natalizumab, $58 \%$ of patients achieved NEDA [67]. The prevalence of JCV seropositivity ranges from 43 to $51 \%$ in pediatric MS cohorts $[67,68]$; therefore, routine monitoring of JCV antibody status is recommended. In recent years, rituximab use in pediatric MS has also increased with similar safety and side effect profiles as the firstline therapies, at least in the short term [65]. A case series of 14 pediatric MS patients receiving rituximab showed no relapses and stable or decreased EDSS scores in 13 of 14 patients [69]. A cohort study of 741 pediatric MS or clinically isolated syndrome patients showed patients who were initially started on newer therapies (fingolimod, dimethyl fumarate, teriflunomide, natalizumab, rituximab, and ocrelizumab) had lower ARRs compared with patients on injectable DMTs (glatiramer acetate or interferon beta) [70]. There are multiple clinical trials in progress studying the use of various DMTs in MS, including the LemKids trial examining the use of alemtuzumab in pediatric patients with relapsing MS [71]. While the potential side effects of higher efficacy DMTs should be considered before starting in the pediatric population, it is worth noting these risks must be weighed against the benefits of reduction in new inflammatory activity and disability progression seen with the newer high-efficacy therapies. Pediatric MS patients have higher annualized relapse rates than adult MS patients [72] and will have a longer duration of disease than in adult-onset MS; therefore, pediatric patients may benefit from early initiation of highefficacy therapies.

Pregnancy has been associated with a lower risk of relapse, particularly in the third trimester [73, 74]. As many women with MS are of childbearing age, pregnancy, contraception, and breastfeeding are important topics for women to discuss with their providers. As the landscape of MS treatment has allowed considering initiation of high-efficacy DMTs earlier in the disease course, the benefits of relapse reductions must be weighed against potential side effects of these more potent therapies, particularly if a patient becomes pregnant. Many of the effective therapies routinely prescribed for treatment of MS can provide deleterious effects on a fetus.

Many DMTs require a washout period between discontinuation of the immunosuppressive agent and conception. Rituximab can cause a transient depletion of $B$ cells in infants born to mothers on this therapy during pregnancy [41]. While there are no existing data on ocrelizumab and B cell lymphopenia in infants, this remains a possible risk given the similar 
mechanism of action on CD20+ cells. It is generally recommended for patients on B cell depleting therapies who wish to become pregnant to continue contraceptive methods for at least 6 months after the last ocrelizumab infusion and 12 months following the last rituximab infusion $[36,41]$, although in practice, many neurologists may advise a shorter window from last infusion to attempts to conceive based on the relatively short half-lives of these medications. The immunosuppressive effects of the anti-CD20 therapies often appear to outlast the half-life of these drugs and can be useful when prescribed for patients who wish to conceive, provided the last dose is far enough in advance to avoid exposure in utero [75]. Cladribine and mitoxantrone are contraindicated for use in pregnancy due to their teratogenic potential, with a recommended washout period of at least 6 months following the last dose before pursuing pregnancy $[56,76]$. Alemtuzumab has a recommended washout period of 6 months, although the immunosuppressive impact of the drug persists even after it is cleared from the serum, allowing for adequate protection from relapses during pregnancy [77]. Natalizumab treatment is typically not recommended during pregnancy and a 2-month washout period is suggested prior to pregnancy. Although, some MS patients with highly active disease have continued on natalizumab throughout pregnancy and in those continuing this therapy through the third trimester have observed reversible hematological abnormalities in the majority of their newborns [78]. Fingolimod may cause fetal harm (two-fold increased risk of congenital malformations) and is not recommended for continuation during pregnancy, with a recommended washout period of at least 2 months [78]. Given the risk of rebound disease activity with natalizumab and fingolimod after a prolonged washout period [77], some clinicians recommend transitioning patients to a different therapy (without such rebound potential) for a couple months prior to attempting to conceive. Although not high-efficacy treatments, glatiramer acetate and interferon therapies do not require a washout period and can be considered up to or sometimes during pregnancy.

With regard to breastfeeding, while the Pregnancy in Multiple Sclerosis (PRIMS) study $(n=227)$ suggested there was no difference in the annualized relapse rate (ARR) between patients who breastfed and those who did not, more recent data suggests breastfeeding could potentially lead to a decrease in the ARR postpartum [73, 79]. One study suggested breastfeeding could be more beneficial in reducing the ARR than resuming a moderately effective DMT within the early postpartum period [79]. A systemic review of 24 studies and meta-analysis of 16 studies encompassing 2974 women with MS demonstrated a 43\% lower rate of postpartum relapse in breastfeeding patients when compared to patients who did not breastfeed [80]. Little is currently known about the effects of DMTs in breast milk, and typically, patients are not restarted on DMTs while breastfeeding, although there are data to suggest this may be too conservative for some therapies. One observational study reviewed $23 \mathrm{MS}$ patients who received monoclonal antibodies (natalizumab, rituximab, and ocrelizumab) during lactation and did not find negative impacts on the development and health of breastfed infants [81]. Another study revealed minimal transfer of rituximab to breast milk with a relative infant dose of less than $0.4 \%$ [82]. This is 
still an area of fervent debate and highlights the importance of individualized discussions between patients and their medical providers regarding breastfeeding and the resumption of DMTs.

\section{Importance of observational and comparative effectiveness studies}

The gold standard for assessing efficacy of a drug is a randomized, double-blind, placebo-controlled clinical trial. However, the inclusion of a placebo arm in today's clinical trials studying the effect of a DMT on disease activity is no longer considered ethical given the abundance of therapies currently available to effectively treat relapsing forms of MS [83]. Hence, the efficacy data of newer DMTs are assessed via active comparator, observational, and comparative effectiveness studies. Retrospective observational studies have suggested that an early highly effective treatment approach is more beneficial in patients with highly active MS; however, the impact of these treatment paradigms on patients with mild to moderately active disease remains unknown. While several notable studies are mentioned in previous sections, there are additional important observational and comparative effectiveness studies worth reviewing. One study using MS registry data from 544 adults with RRMS used propensity score matching to compare patients receiving high-efficacy therapy (rituximab, ocrelizumab, mitoxantrone, alemtuzumab, or natalizumab) early (within 2 years) versus late (within 4-6 years) with respect to their first clinical event

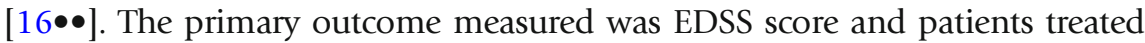
with high-efficacy DMTs within 2 years of clinical presentation had lower EDSS scores than patients treated later in the disease course. A cohort study spanning 19 years and comprising 592 patients evaluated patients treated with early intensive treatments (alemtuzumab and natalizumab) compared with patients treated with an escalation approach to DMTs (interferons, glatiramer acetate, dimethyl fumarate, fingolimod, and teriflunomide) [22•], with a primary outcome of change in EDSS score over 5 years. Patients treated with an escalation approach showed a higher increase in EDSS score over 5 years $($ mean +1.2$)$ compared to patients receiving early intensive treatments (mean +0.3$)$, even after adjusting for age, sex, year of starting DMT, and escalating to high-efficacy treatments while in the escalation group. There was no significant difference between groups in the secondary outcome, sustained accumulation of disability over at least 6 months.

Advances in neuroimaging and paraclinical data have important implications for research in MS, as these data can further delineate disease status. One observational cohort study compared MRI imaging data (whole brain, subcortical gray matter, cortical gray matter, and cerebral white matter volume fractions) in $78 \mathrm{MS}$ patients grouped by higher efficacy (natalizumab or rituximab) or lower efficacy (IFN- $\beta$ or GA) DMTs. Patients on higher efficacy DMTs were found to have slower rates of subcortical atrophy, specifically in the thalamus and putamen [84]. Other recent studies have demonstrated a more robust impact on decreasing neurofilament light chain levels and lessening the rate 
of ganglion cell inner plexiform layer loss on optical coherence tomography with natalizumab compared to interferons and/or glatiramer acetate $[85,86]$.

While observational studies can provide important insight into the realworld effectiveness of a DMT, they have limitations that are important to recognize. Many observational studies include small sample sizes, making results difficult to generalize to the broader population of MS patients. Often, data from observational studies are extracted from MS patient registries that consist predominantly of unblinded clinical measures such as relapses or the EDSS score, and largely exclude imaging and other important paraclinical data. Incorporating these data points in future studies is imperative, as clinicians often make decisions to escalate therapy based on radiologic disease activity and treating patients before clinical symptoms emerge is imperative to preventing future disability. Additionally, while propensity score matching aims to eliminate some confounding variables when studying DMTs and treatment paradigms, the lack of randomization cannot eliminate variables such as clinician prescribing habits and confounding by indication. Finally, these studies do not take a systematic approach to directly test treatment strategies of early intensive or escalation approaches.

Two pivotal clinical trials currently taking place aiming to answer this question regarding treatment paradigms are the TREAT-MS (TRaditional versus Early Aggressive Therapy for MS) trial and the DELIVER-MS (Determining the Effectiveness of earLy Intensive Versus Escalation approaches for the treatment of Relapsing-remitting MS) trial. The primary endpoint in TREAT-MS is time to sustained disability progression, while the primary endpoint in DELIVER-MS is normalized whole brain volume loss. Table 1 provides an overview of the study design and outcomes for TREAT-MS and DELIVER-MS clinical trials.

With the expansion of multiple highly effective therapeutics to treat relapsing forms of MS, a shift in the treatment paradigm has occurred in which individuals with MS are being treated earlier with highly effective DMTs. With recently approved agents showing more robust reductions in new inflammatory activity over older agents, many patients are now being placed on highly efficacious DMTs at initial diagnosis, rather than opting for an escalation treatment approach. The TREAT-MS and DELIVER-MS trials aim to evaluate whether an early aggressive versus escalation treatment approach differentially impacts future disability. Progression of disability in patients with MS can not only have major implications in quality of life and functional status, but also result in decreased work productivity [87]. Therefore, understanding if early intensive treatment of MS at time of diagnosis truly slows the accrual of disability that occurs from clinical relapses and ongoing neuroinflammation, and whether this is the case across all people with MS or only those with certain early disease features, is critical. While newly approved agents have mostly shown limited significant risks in the short term, longitudinal studies are imperative to determining the long-term risk profiles associated with the highly effective DMTs.

Future directions of MS therapeutics include development of more therapies that are effective in treating progressive MS and those that impact the innate immune system. Additionally, there is a huge unmet need for neurorepair and 
Table 1. Overview of the study design and outcomes for TREAT-MS and DELIVER-MS clinical trials

TREAT-MS (TRaditional versus Early Aggressive Therapy for MS)

\begin{tabular}{|c|c|}
\hline $\begin{array}{l}\text { Intervention/treatment } \\
\text { groups }\end{array}$ & $\begin{array}{l}\text { Early aggressive therapies } \\
\text { - Natalizumab } \\
\text { - Alemtuzumab } \\
\text { - Ocrelizumab } \\
\text { - Rituximab } \\
\text { - Ofatumumab } \\
\text { - Cladribine } \\
\text { Traditional therapies } \\
\text { - Subcutaneous, intramuscular, and pegylated } \\
\text { interferon } \\
\text { - Glatiramer acetate } \\
\text { - Teriflunomide } \\
\text { - Dimethyl fumarate, diroximel fumarate } \\
\text { - Fingolimod, siponimod, ozanimod }\end{array}$ \\
\hline Group assignment & $\begin{array}{l}\text { Participant stratification for higher versus } \\
\text { lower risk for long-term disability, then 1:1 } \\
\text { randomization within each disability risk } \\
\text { stata }(n=900)\end{array}$ \\
\hline Primary outcome & $\begin{array}{l}\text { Time to sustained disability progression using } \\
\text { blinded EDSS plus }\end{array}$ \\
\hline Secondary outcomes & $\begin{array}{l}\text { Timeframe: up to } 63 \text { months } \\
\text { - PDDS } \\
\text { - Blinded MSFC (composite and individual) } \\
\text { - LCLA } \\
\text { - Patient-reported incomplete relapse recovery } \\
\text { - Neurologic exam-based incomplete relapse } \\
\text { recovery } \\
\text { - SDMT } \\
\text { - MSIS-29 } \\
\text { - Neuro-QoL } \\
\text { - Employment status } \\
\text { - Marital status } \\
\text { - Serious adverse events } \\
\text { - Adverse events resulting in a decision to } \\
\text { disease-modifying therapy discontinuation/- } \\
\text { change }\end{array}$ \\
\hline Other outcomes & $\begin{array}{l}\text { - Compare changes in whole brain and } \\
\text { normalized gray matter volumes, cortical } \\
\text { thickness, and subcortical gray matter } \\
\text { compartment volumes, and measures of T2 } \\
\text { lesion burden } \\
\text { - Number of relapses } \\
\text { - Number of new brain lesions on MRI }\end{array}$ \\
\hline
\end{tabular}

DELIVER-MS (Determining the Effectiveness of earLy Intensive Versus Escalation Approaches for the treatment of Relapsing-remitting MS)

Early highly effective therapies

- Natalizumab

- Alemtuzumab

- Ocrelizumab

- Rituximab

- Ofatumumab

Escalation therapies

- Beta interferon

- Glatiramer acetate

- Teriflunomide

- Dimethyl fumarate, diroximel fumarate

- Fingolimod, siponimod, ozanimod

- Cladribine

Cohort A ( $n=400)$ : 1:1 randomization Cohort B $(n=400)$ : observational cohort (participants not willing to be randomized)

Normalized whole brain volume loss using MRI from baseline to month 36

- Normalized whole brain volume loss using MRI from month 6 to month 36

- Proportion of patients with worsening in multidimensional composite confirmed over 12 months (EDSS, T25FW, 9HPT, SDMT, LCLA)

- Change in MSIS-29 from baseline to month 36

- Change in Neuro-QoL from baseline to month 36

- Serious adverse events

- Percentage of any $\mathrm{SAE}$, grade 3 and $4 \mathrm{AE}$, and AEs that lead to treatment discontinuation

- TSQM

- Brain volume loss from baseline to month 12 , 12 to 24,12 to 36 , and months 24 to 36 - Compare changes in T2 lesions volume, T1 hypointense lesion volume, and gray matter fraction 
Table 1. (Continued)

\section{TREAT-MS (TRaditional versus Early Aggressive Therapy for MS)}

- Compare change in retinal nerve fiber layer and/or ganglion cell inner plexiform layer thickness on OCT

- Number of new medications, escalated doses of medications, and non-pharmacologic interventions for MS-related symptoms

Substudy
- Biorepository for biomarker discovery studies

- COVID-19-related enhancement study

\section{DELIVER-MS (Determining the Effectiveness of earLy Intensive Versus Escalation Approaches for the treatment of Relapsing-remitting MS)}

- Biorepository for biomarker discovery studies

EDSS plus: a composite endpoint that includes EDSS change OR 20\% worsening on either the timed 25-ft walk test or the nine-hole peg test that is sustained 6 months later

PDDS patient-determined disease steps, MSFC Multiple Sclerosis Functional Composite score, T25FWT timed 25-foot walk test, 9HPT nine-hole peg test, PASAT Paced Auditory Serial Addition Test, LCLA low contrast letter acuity, SDMT Symbol Digit Modality Test, MSIS-29 Multiple Sclerosis Impact Scale, Neuro-QoL Quality of Life in Neurologic Disorders, OCT optical coherence tomography, SAE serious adverse event, $A E$ adverse event, TSQM Treatment Satisfaction Questionnaire for Medication

neuroprotective therapies. While these therapies do not yet exist, various agents are being tested in clinical trials providing hope for interventions that go beyond suppressing inflammation. Finally, little is currently known regarding management of late-onset relapsing MS and use of higher efficacy DMTs in patients greater than 55 years old due to the current design of clinical trials.

The landscape of MS treatment has changed dramatically since the approval of the first DMT in the early 1990s. The change in current treatment paradigms and ongoing basic science and translational research leading to clinical trials addressing novel therapeutic agents will undeniably result in remarkable continued advances in this field.

\section{References and Recommended Reading}

Papers of particular interest, published recently, have been

highlighted as:

- Of importance

$\bullet$ Of major importance

1. Alroughani R, Akhtar S, Ahmed S, Behbehani R, AlHashel J. Is time to reach EDSS 6.0 faster in patients with late-onset versus young-onset multiple sclerosis? PLoS One. 2016;11(11):e0165846.

2. Cree BAC, Khan O, Bourdette D, Goodin DS, Cohen JA, Marrie RA, et al. Clinical characteristics of African Americans vs Caucasian Americans with multiple sclerosis. Neurology. 2004;63(11):2039-45.

3. Ventura RE, Antezana AO, Bacon T, Kister I. Hispanic Americans and African Americans with multiple sclerosis have more severe disease course than Caucasian Americans. Mult Scler. 2017;23(11):1554-7.

4. Deloire M, Ruet A, Hamel D, Bonnet M, Brochet B. Early cognitive impairment in multiple sclerosis predicts disability outcome several years later. Mult Scler. 2010;16(5):581-7.

5. Scott TF, Schramke CJ. Poor recovery after the first two attacks of multiple sclerosis is associated with poor outcome five years later. J Neurol Sci. 2010;292(12):52-6. 
6. Mowry EM, Pesic M, Grimes B, Deen S, Bacchetti P, Waubant E. Demyelinating events in early multiple sclerosis have inherent severity and recovery. Neurology. 2009;72(7):602-8.

7. Scott TF, Schramke CJ, Novero J, Chieffe C. Short-term prognosis in early relapsing-remitting multiple sclerosis. Neurology. 2000;55(5):689-93.

8. Tintore M, Rovira À, Río J, Otero-Romero S, Arrambide G, Tur C, et al. Defining high, medium and low impact prognostic factors for developing multiple sclerosis. Brain. 2015;138(Pt 7):1863-74.

9. Brex PA, Ciccarelli O, O'Riordan JI, Sailer M, Thompson AJ, Miller DH. A longitudinal study of abnormalities on MRI and disability from multiple sclerosis. N Engl J Med. 2002;346(3):158-64.

10. Kupersmith MJ, Anderson S, Kardon R. Predictive value of 1 month retinal nerve fiber layer thinning for deficits at 6 months after acute optic neuritis. Mult Scler. 2013;19(13):1743-8.

11. Leray E, Coustans M, Le Page E, Yaouanq J, Oger J, Edan G. 'Clinically definite benign multiple sclerosis', an unwarranted conceptual hodgepodge: evidence from a 30-year observational study. Mult Scler. 2013;19(4):458-65.

12. Prosperini L, Mancinelli CR, Solaro CM, Nociti V, Haggiag S, Cordioli C, et al. Induction versus escalation in multiple sclerosis: a 10-year real world study. Neurotherapeutics. 2020;31.

13. Banwell B, Ben-Zacharia A, Bowen J, Therapeutics T, Cohen B, Cree B, et al. DISCLOSURES 2019 Revision-Writing Team Kathleen Costello, MS, ANP-BC, MSCN - nothing to disclose Rosalind Kalb, PhD - Consultant: Sanofi-Genzyme and Novartis Pharmaceuticals.: 72.

14. Scalfari A, Neuhaus A, Degenhardt A, Rice GP, Muraro PA, Daumer M, et al. The natural history of multiple sclerosis, a geographically based study 10: relapses and long-term disability. Brain. 2010;133(7):1914-29.

15. Jokubaitis VG, Spelman T, Kalincik T, Lorscheider J, Havrdova E, Horakova D, et al. Predictors of long-term disability accrual in relapse-onset multiple sclerosis. Ann Neurol. 2016;80(1):89-100.

16.• He A, Merkel B, Brown JWL, Zhovits Ryerson L, Kister I, Malpas CB, et al. Timing of high-efficacy therapy for multiple sclerosis: a retrospective observational cohort study. Lancet Neurol. 2020;19(4):307-1.

Demonstrates that using high-efficacy therapies earlier in the disease course may result in less long-term disability compared to using them later in the course of the disease.

17. Castillo-Trivino T, Mowry EM, Gajofatto A, Chabas D, Crabtree-Hartman E, Cree BA, et al. Switching multiple sclerosis patients with breakthrough disease to secondline therapy. PLoS One [Internet]. 2011;6(2):3401 Available from: https://www.ncbi.nlm.nih.gov/pmc/ articles/PMC3033401/.

18. Giovannoni G, Butzkueven $H$, Dhib-Jalbut $S$, Hobart J, Kobelt G, Pepper G, et al. Brain health: time matters in multiple sclerosis. Multi Scleros Relat Disorders. 2016;9:S5-48.
19. Brown JWL, Coles A, Horakova D, Havrdova E, Izquierdo $\mathrm{G}$, Prat $\mathrm{A}$, et al. Association of initial diseasemodifying therapy with later conversion to secondary progressive multiple sclerosis. JAMA. 2019;321(2):175-87.

20. Kalincik T, Horakova D, Spelman T, Jokubaitis V, Trojano M, Lugaresi A, et al. Switch to natalizumab versus fingolimod in active relapsing-remitting multiple sclerosis. Ann Neurol. 2015;77(3):425-35.

21. Lizak N, Lugaresi A, Alroughani R, Lechner-Scott J, Slee M, Havrdova E, et al. Highly active immunomodulatory therapy ameliorates accumulation of disability in moderately advanced and advanced multiple sclerosis. J Neurol Neurosurg Psychiatry. 2017;88(3):196-203.

22.• Harding K, Williams O, Willis M, Hrastelj J, Rimmer A, Joseph F, et al. Clinical outcomes of escalation vs early intensive disease-modifying therapy in patients with multiple sclerosis. JAMA Neurol. 2019;76(5):536-4.

More favorable outcomes are seen in the intermediate term with an early aggressive treatment approach as compared to an escalation treatment approach.

23. Hauser SL, Bar-Or A, Cohen JA, Comi G, Correale J, Coyle PK, et al. Ofatumumab versus teriflunomide in multiple sclerosis. N Engl J Med [Internet]. 2020; [cited 2020 Sep 18]; Available from: https://www-nejm-org. proxy1.library.jhu.edu/doi/10.1056/ NEJMoa1917246.

24. Ryerson LZ, Foley J, Chang I, Kister I, Cutter G, Metzger $\mathrm{RR}$, et al. Risk of natalizumab-associated PML in patients with MS is reduced with extended interval dosing. Neurology. 2019;93(15):e1452-62.

25. Polman $\mathrm{CH}, \mathrm{O}^{\prime}$ Connor PW, Havrdova E, Hutchinson M, Kappos L, Miller DH, et al. A randomized, placebocontrolled trial of natalizumab for relapsing multiple sclerosis. N Engl J Med. 2006;354(9):899-910.

26. Butzkueven H, Licata S, Jeffery D, Arnold DL, Filippi M, Geurts JJ, et al. Natalizumab versus fingolimod for patients with active relapsing-remitting multiple sclerosis: results from REVEAL, a prospective, randomised head-to-head study. BMJ Open. 2020;10(10):60 Available from: https://www.ncbi.nlm.nih.gov/pmc/ articles/PMC7577060/.

27. Spelman T, Kalincik T, Jokubaitis V, Zhang A, Pellegrini $\mathrm{F}$, Wiendl $\mathrm{H}$, et al. Comparative efficacy of first-line natalizumab vs IFN- $\beta$ or glatiramer acetate in relapsing MS. Neurol Clin Pract. 2016;6(2):102-15.

28. Prosperini L, Saccà F, Cordioli C, Cortese A, Buttari F, Pontecorvo S, et al. Real-world effectiveness of natalizumab and fingolimod compared with selfinjectable drugs in non-responders and in treatmentnaïve patients with multiple sclerosis. J Neurol. 2017;264(2):284-94.

29. Spelman T, Kalincik T, Zhang A, Pellegrini F, Wiendl H, Kappos L, et al. Comparative efficacy of switching to natalizumab in active multiple sclerosis. Ann Clin Transl Neurol. 2015;2(4):373-87.

30.• Luna G, Alping P, Burman J, Fink K, Fogdell-Hahn A, Gunnarsson $M$, et al. Infection risks among patients with multiple sclerosis treated with fingolimod, 
natalizumab, rituximab, and injectable therapies.

JAMA Neurol. 2020;77(2):184-9.

This is an extremely important study as it sheds light on the differential impact of immune therapies causing serious infections over time. These data are important to keep in mind when assessing the long-term risk-benefit profiles of treatment strategies.

31. Tysabri (natalizumab) [package insert]. [Internet]. [cited 2020 Apr 30]. Available from: https://www. accessdata.fda.gov/drugsatfda_docs/label/2012/ 125104s0576lbl.pdf

32. Hauser SL, Arnold DL, Fox RJ, Sarkar N, Smith CH. B cell depletion with rituximab in relapsing-remitting multiple sclerosis. N Engl J Med. 2008;13.

33. Hawker K, O'Connor P, Freedman MS, Calabresi PA, Antel J, Simon J, et al. Rituximab in patients with primary progressive multiple sclerosis: results of a randomized double-blind placebo-controlled multicenter trial. Ann Neurol. 2009;66(4):460-71.

34. Alping P, Frisell T, Novakova L, Islam-Jakobsson P, Salzer J, Björck A, et al. Rituximab versus fingolimod after natalizumab in multiple sclerosis patients. Ann Neurol. 2016;79(6):950-8.

35. Granqvist $\mathrm{M}$, Boremalm $\mathrm{M}$, Poorghobad A, Svenningsson A, Salzer J, Frisell T, et al. Comparative effectiveness of rituximab and other initial treatment choices for multiple sclerosis. JAMA Neurol. 2018;75(3):320-7.

36. OCREVUSTM (ocrelizumab) injection [package insert].: 18 .

37. Hauser SL, Bar-Or A, Comi G, Giovannoni G, Hartung H-P, Hemmer B, et al. Ocrelizumab versus interferon beta-1a in relapsing multiple sclerosis [Internet]. https://doi.org/10.1056/NEJMoa1601277. Massachusetts Medical Society; 2016 [cited 2020 Apr 29]. Available from: https://www.nejm.org/doi/10.1056/ NEJMoa1601277?url_ver=Z39.88-2003\&rfr_id=ori\% 3Arid\%3Acrossref.org\&rfr_dat=cr_pub\%3Dwww.ncbi. nlm.nih.gov

38. Montalban X, Hauser SL, Kappos L, Arnold DL, Bar-Or A, Comi G, et al. Ocrelizumab versus placebo in primary progressive multiple sclerosis. $\mathrm{N}$ Engl J Med. 2017;376(3):209-20.

39. Rommer PS, Dudesek A, Stüve O, Zettl UK. Monoclonal antibodies in treatment of multiple sclerosis. Clin Exp Immunol. 2014;175(3):373-84.

40. Nguyen A, Gresle M, Marshall T, Butzkueven H, Field J. Monoclonal antibodies in the treatment of multiple sclerosis: emergence of B cell-targeted therapies. Br J Pharmacol. 2017

Jul;174(13):1895-907.

41. Rituxan (rituximab) injection [package insert]. [Internet]. [cited 2020 May 20]. Available from: https:// www.accessdata.fda.gov/drugsatfda_docs/label/2012/ 103705s5367s5388lbl.pdf

42. Mayer L, Kappos L, Racke MK, Rammohan K, Traboulsee A, Hauser SL, et al. Ocrelizumab infusion experience in patients with relapsing and primary progressive multiple sclerosis: results from the phase 3 randomized OPERA I, OPERA II, and ORATORIO studies. Multi Scleros Relat Disorders. 2019;30:236-43.

43. Clifford DB, Ances B, Costello C, Rosen-Schmidt S, Andersson M, Parks D, et al. Rituximab-associated progressive multifocal leukoencephalopathy in rheumatoid arthritis. Arch Neurol. 2011;68(9):1156-64.

44. Sormani MP. An Italian programme for COVID-19 infection in multiple sclerosis. Lancet Neurol. 2020;19(6):481-2.

45. Cohen JA, Coles AJ, Arnold DL, Confavreux C, Fox EJ, Hartung H-P, et al. Alemtuzumab versus interferon beta $1 \mathrm{a}$ as first-line treatment for patients with relapsing-remitting multiple sclerosis: a randomised controlled phase 3 trial. Lancet. 2012;380(9856):1819-28.

46. Coles AJ, Twyman CL, Arnold DL, Cohen JA, Confavreux C, Fox EJ, et al. Alemtuzumab for patients with relapsing multiple sclerosis after diseasemodifying therapy: a randomised controlled phase 3 trial. Lancet. 2012;380(9856):1829-39.

47. Kalincik T, Brown JWL, Robertson N, Willis M, Scolding N, Rice CM, et al. Treatment effectiveness of alemtuzumab compared with natalizumab, fingolimod, and interferon beta in relapsing-remitting multiple sclerosis: a cohort study. Lancet Neurol. 2017;16(4):271-81.

48. Lemtrada (alemtuzumab) injection [package insert]. [Internet]. [cited 2020 May 20]. Available from: https:// www.accessdata.fda.gov/drugsatfda_docs/label/2017/ 103948s5158lbl.pdf

49. Millefiorini E, Gasperini C, Pozzilli C, D'Andrea F, Bozzao A, Calo A, et al. Randomized placebocontrolled trial of mitoxantrone in relapsing-remitting multiple sclerosis: 24-month clinical and MRI outcome. J Neurol. 1997;244(3):153-9.

50. Hartung H-P, Gonsette R, Konig N, Kwiecinski H, Guseo A, Morrissey SP, et al. Mitoxantrone in progressive multiple sclerosis: a placebo-controlled, doubleblind, randomised, multicentre trial. Lancet. 2002;360(9350):2018-25.

51. Novantrone (mitoxantrone) injection [package insert]. [Internet]. [cited 2020 May 20]. Available from: https:// www.accessdata.fda.gov/drugsatfda_docs/label/2012/ 019297s035lbl.pdf

52. Giovannoni G, Comi G, Cook S, Rammohan K, Rieckmann P, Soelberg Sørensen P, et al. A placebocontrolled trial of oral cladribine for relapsing multiple sclerosis. N Engl J Med. 2010;362(5):416-26.

53. Giovannoni G, Soelberg Sorensen P, Cook S, Rammohan KW, Rieckmann P, Comi G, et al. Efficacy of cladribine tablets in high disease activity subgroups of patients with relapsing multiple sclerosis: a post hoc analysis of the CLARITY study. Multi Scleros. 2019;25(6):819-27.

54. Signori A, Saccà F, Lanzillo R, Maniscalco GT, Signoriello E, Repice AM, et al. Cladribine vs other drugs in MS: merging randomized trial with real-life data. Neurol Neuroimmunol Neuroinflam. 2020;7(6). 
55. Berardi A, Siddiqui MK, Treharne C, Harty G, Wong SL. Estimating the comparative efficacy of cladribine tablets versus alternative disease modifying treatments in active relapsing-remitting multiple sclerosis: adjusting for patient characteristics using meta-regression and matching-adjusted indirect treatment comparison approaches. Curr Med Res Opin. 2019;35(8):1371-8.

56. Mavenclad (cladribine) tablets [package insert]. [Internet]. [cited 2020 May 20]. Available from: https:// www.accessdata.fda.gov/drugsatfda_docs/label/2019/ 022561s000lbl.pdf

57. Das J, Sharrack B, Snowden JA. Autologous haematopoietic stem cell transplantation in multiple sclerosis: a review of current literature and future directions for transplant haematologists and oncologists. Curr Hematol Malig Rep. 2019;14(2):127-35.

58. Muraro PA, Pasquini M, Atkins H, Bowen J, Farge D, Fassas A, et al. Long term outcomes after autologous hematopoietic stem cell transplantation for multiple sclerosis. JAMA Neurol. 2017;74(4):459-69.

59. Cohen JA, Baldassari LE, Atkins HL, Bowen JD, Bredeson C, Carpenter PA, et al. Autologous hematopoietic cell transplantation for treatment-refractory relapsing multiple sclerosis: position statement from the American Society for Blood and Marrow Transplantation. Biol Blood Marrow Transplant. 2019;25(5):84554.

60. Lee H, Narayanan S, Brown RA, Chen JT, Atkins HL, Freedman MS, et al. Brain atrophy after bone marrow transplantation for treatment of multiple sclerosis. Mult Scler. 2017;23(3):420-31.

61. Best Available Therapy Versus Autologous Hematopoetic Stem Cell Transplant for Multiple Sclerosis (BEAT-MS) - Full Text View - ClinicalTrials.gov [Internet]. [cited 2020 Jul 31]. Available from: https:// clinicaltrials.gov/ct2/show/NCT04047628

62. Fox E, Lovett-Racke AE, Gormley M, Liu Y, Petracca M, Cocozza $S$, et al. A phase 2 multicenter study of ublituximab, a novel glycoengineered anti-CD20 monoclonal antibody, in patients with relapsing forms of multiple sclerosis. Mult Scler.

2020;30:1,352,458,520,918,375

63. Montalban X, Arnold DL, Weber MS, Staikov I, Piasecka-Stryczynska K, Willmer J, et al. Placebocontrolled trial of an oral BTK inhibitor in multiple sclerosis. N Engl J Med. 2019;380(25):2406-17.

64. Waldman AT, Gorman MP, Rensel MR, Austin TE, Hertz DP, Kuntz NL. Management of pediatric central nervous system demyelinating disorders: consensus of United States neurologists. J Child Neurol.

2011;26(6):675-82.

65. Krysko KM, Graves J, Rensel M, Weinstock-Guttman B, Aaen G, Benson L, et al. Use of newer diseasemodifying therapies in pediatric multiple sclerosis in the US. Neurology. 2018;91(19):e1778-87.

66. Chitnis T, Arnold DL, Banwell B, Brück W, Ghezzi A, Giovannoni $G$, et al. Trial of fingolimod versus interferon beta-1a in pediatric multiple sclerosis. New England Journal of Medicine [Internet]. 2018 Sep 12 [cited 2021 Feb 25]; Available from: https://www. nejm.org/doi/10.1056/NEJMoa1800149

67. Ghezzi A, Moiola L, Pozzilli C, Brescia-Morra V, Gallo $\mathrm{P}$, Grimaldi LME, et al. Natalizumab in the pediatric MS population: results of the Italian registry. BMC Neurol [Internet]. 2015;15 Available from: https:// www.ncbi.nlm.nih.gov/pmc/articles/PMC4583752/.

68. Huppke P, Hummel H, Ellenberger D, Pfeifenbring S, Stark W, Huppke B, et al. JC virus antibody status in a pediatric multiple sclerosis cohort: prevalence, conversion rate and influence on disease severity. Mult Scler. 2015;21(4):382-7.

69. Salzer J, Lycke J, Wickström R, Naver H, Piehl F, Svenningsson A. Rituximab in paediatric onset multiple sclerosis: a case series. J Neurol. 2016;263(2):322-6.

70. Krysko KM, Graves JS, Rensel M, Weinstock-Guttman B, Rutatangwa A, Aaen G, et al. Real-world effectiveness of initial disease-modifying therapies in pediatric multiple sclerosis. Ann Neurol. 2020;88(1):42-55.

71. Genzyme, a Sanofi Company. A multi-center, openlabel, single-arm, before and after switch study to evaluate the efficacy, safety and tolerability of alemtuzumab in paediatric patients with relapsing remitting multiple sclerosis (RRMS) With disease activity on prior disease modifying therapy (DMT) [Internet]. clinicaltrials.gov; 2020 May [cited 2020 Jul 31]. Report No.: NCT03368664. Available from: https:// clinicaltrials.gov/ct2/show/NCT03368664

72. Gorman MP, Healy BC, Polgar-Turcsanyi M, Chitnis T. Increased relapse rate in pediatric-onset compared with adult-onset multiple sclerosis. Arch Neurol [Internet]. 2009;66(1) Available from: http://archneur. jamanetwork.com/article.aspx?doi=10.1001/ archneurol.2008.505.

73. Vukusic S, Hutchinson M, Hours M, Moreau T, Cortinovis-Tourniaire P, Adeleine P, et al. Pregnancy and multiple sclerosis (the PRIMS study): clinical predictors of post-partum relapse. Brain. 2004;127(Pt 6):1353-60.

74. Hughes SE, Spelman T, Gray OM, Boz C, Trojano M, Lugaresi A, et al. Predictors and dynamics of postpartum relapses in women with multiple sclerosis. Mult Scler. 2014;20(6):739-46.

75. Gelfand JM, Cree BAC, Hauser SL. Ocrelizumab and other CD20+ B Cell-depleting therapies in multiple sclerosis. Neurotherapeutics. 2017;14(4):835-41.

76. Vukusic S, Marignier R. Multiple sclerosis and pregnancy in the "treatment era.". Nat Rev Neurol. 2015;11(5):280-9.

77. Alroughani R, Akhtar S, Zeineddine M, El Kouzi Y, El Ayoubi NK, Ahmed SF, et al. Risk of relapses during pregnancy among multiple sclerosis patients. Mult Scler Relat Disord. 2019;34:9-13.

78. Canibaño B, Deleu D, Mesraoua B, Melikyan G, Ibrahim F, Hanssens Y. Pregnancy-related issues in women with multiple sclerosis: an evidence-based review with practical recommendations. J Drug Assess. 2020;9(1):20-36. https://doi.org/10.1080/21556660. 2020.1721507. 
79. Langer-Gould A, Smith JB, Albers KB, Xiang AH, Wu J, Kerezsi EH, et al. Pregnancy-related relapses and breastfeeding in a contemporary multiple sclerosis COhort. Neurology. 2020;94(18):e1939-49.

80. Krysko KM, Rutatangwa A, Graves J, Lazar A, Waubant E. Association between breastfeeding and postpartum multiple sclerosis relapses. JAMA Neurol. 2020;77(3):327-38.

81. Ciplea AI, Langer-Gould A, de Vries A, Schaap T, Thiel $S$, Ringelstein $M$, et al. Monoclonal antibody treatment during pregnancy and/or lactation in women with MS or neuromyelitis optica spectrum disorder. Neurol Neuroimmunol Neuroinflamm [Internet]. 2020;7(4). Available from: https://www.ncbi.nlm.nih.gov/pmc/ articles/PMC7188475/

82. Krysko KM, LaHue SC, Anderson A, Rutatangwa A, Rowles W, Schubert RD, et al. Minimal breast milk transfer of rituximab, a monoclonal antibody used in neurological conditions. Neurol Neuroimmunol Neuroinflamm [Internet]. 2019;7(1) Available from: https://www.ncbi.nlm.nih.gov/pmc/articles/ PMC6857908/.

83. Zhang Y, Salter A, Wallström E, Cutter G, Stüve O. Evolution of clinical trials in multiple sclerosis. Ther Adv Neurol Disord [Internet]. 2019;12 Available from: https://www.ncbi.nlm.nih.gov/pmc/articles/ PMC6391540/.

84. Sotirchos ES, Gonzalez-Caldito N, Dewey BE, Fitzgerald KC, Glaister J, Filippatou A, et al. Effect of disease- modifying therapies on subcortical gray matter atrophy in multiple sclerosis. Mult Scler. 2019;11:1,352,458,519,826,364.

85. Button J, Al-Louzi O, Lang A, Bhargava P, Newsome $\mathrm{SD}$, Frohman T, et al. Disease-modifying therapies modulate retinal atrophy in multiple sclerosis. Neurology. 2017;88(6):525-32.

86. Calabresi PA. Serum neurofilament light (NfL) for disease prognosis and treatment monitoring in multiple sclerosis patients: is it ready for implementation into clinical care? [Internet]. Available from: https:// onlinelibrary.ectrims-congress.eu/ectrims/2018/ ectrims-2018/231907/peter.calabresi.serum. neurofilament.light.\%28nfl\%29.for.disease.prognosis. and.html

87. Chen J, Taylor BV, Blizzard L, Simpson S, Palmer AJ, van der Mei IAF. Effects of multiple sclerosis diseasemodifying therapies on employment measures using patient-reported data. J Neurol Neurosurg Psychiatry. 2018;89(11):1200-7.

\section{Publisher's Note}

Springer Nature remains neutral with regard to jurisdictional claims in published maps and institutional affiliations. 